\title{
Préparation de phosphocaséinate natif par microfiltration sur membrane
}

\author{
A Pierre, J Fauquant, Y Le Graet, M Piot, JL Maubois \\ INRA, Laboratoire de recherches de technologie laitière, 65, rue de Saint-Brieuc, \\ 35042 Rennes Cedex, France
}

(Reçu le 20 mai 1992; accepté le 17 juin 1992)

\begin{abstract}
Résumé - Le phosphocaséinate natif (PPCN) était séparé du lait cru par microfiltration tangentielle sur membrane (diamètre des pores : $0,2 \mu \mathrm{m}$ ) suivie d'une purification par diafiltration avec de l'eau. Le suivi analytique des liquides obtenus de part et d'autre de la membrane montrait une perméation conforme aux lois de la filtration des protéines majeures du lactosérum ( $\beta$-lactoglobuline et $\alpha$-lactalbumine), du lactose et des sels minéraux solubles et au contraire, une rétention élevée des composants solubles à haut poids moléculaire ainsi que des protéoses-peptones. Le rétentat final était séché par atomisation. Ses teneurs en matière azotée $(N \times 6,38)$ et en cendres étaient respectivement de $89 \%$ et de $9 \%$ par rapport à la substance sèche. L'aptitude à la coagulation par la présure, de la solution reconstituée a été comparée à celle du lait cru. Le temps de coagulation est réduit de $53 \%$; la cinétique d'organisation du gel (appréciée par le $K_{20}$ ) et la fermeté finale (appréciée à $30 \mathrm{~min}$ ) sont également accrues de plus de $50 \%$. La propriété de former un gel sous l'action de la présure après un traitement thermique de $100{ }^{\circ} \mathrm{C}-5$ min est conservée, contrairement au lait qui, soumis au même traitement, ne coagule plus.
\end{abstract}

microfiltration / phosphocaséinate / coagulation présure / traitement thermique

Summary - Native micellar casein separation through cross flow membrane microfiltration. Native phosphocaseinate (PPCN) was separated from raw milk by tangential membrane microfiltration (pore diameter: $0.2 \mu \mathrm{m}$ ) followed by purification through water diafiltration. Analytical survey of both liquids issued by membrane treatment showed permeation rates matching mathematical laws for the main whey proteins ( $\beta$-lactoglobulin and $\alpha$-lactalbumin), lactose and soluble mineral salts. On the contrary, a high retention of high molecular weight soluble components and proteose-peptone fraction was observed. Final diafiltrated retentate was spray-dried. Its protein $(N \times 6.38)$ and ash contents in total solids were respectively $89 \%$ and $9 \%$. Rennet coagulating ability of PPCN reconstituted solution was compared to that of raw milk. Coagulation time was reduced by $53 \%$; gel development kinetics (estimated by $\mathrm{K}_{20}$ ) and final firmness (estimated at $30 \mathrm{~min}$ ) were increased by more than $50 \%$. When a severe heat treatment $\left(100^{\circ} \mathrm{C}-5 \mathrm{~min}\right)$ was applied, milk lost its ability to coagulate after rennet action; PPCN solution did not, but its coagulation time was increased and the curd was slightly weaker. 


\section{INTRODUCTION}

Les procédés industriels de séparation de la caséine mettent en œuvre une déstabilisation de ces protéines, soit par abaissement du $\mathrm{pH}$ du lait au point isoélectrique, soit par hydrolyse enzymatique de la caséine $\kappa$, composant stabilisant les micelles. Les formes d'utilisation des caséines ou paracaséines, insolubles, ainsi obtenues étant relativement restreintes, il est procédé, en général à l'addition de divers alcalis (caséines) ou de séquestrants du calcium (paracaséines) pour obtenir des produits solubles répondant aux besoins très divers des industries utilisatrices (Mulvihill et Fox, 1989). Les caséinates industriels ainsi produits, diffèrent dans leur composition fine (teneur en minéraux colloîdaux notamment) et dans leurs structures tertiaire et quaternaire, de la forme native micellaire des caséines. II est donc probable que leurs propriétés fonctionnelles initiales aient été profondément modifiées.

Dans le lait, l'essentiel de la caséine : 90 à $95 \%$ (Alais, 1984; Holt, 1985) se trouve sous forme de particules ayant un diamètre compris entre 20 et $200 \mathrm{~nm}$. Une séparation basée sur la différence de masses volumiques est aisée à réaliser au niveau du laboratoire mais l'extrapolation industrielle, combinant ultracentrifugation et ultrafiltration, proposée par Brulé et al (1979) n'a pas connu le développement espéré en raison de contraintes insurmontables au niveau du dispositif d'évacuation continue du sédiment (Maubois, 1984). Dans une étude récente, Fauquant et al (1988) ont démontré que la mise en contact du lait écrémé avec une membrane de microfiltration ayant un diamètre moyen de pores de $0,2 \mu \mathrm{m}$ conduisait à une rétention sélective de la caséine micellaire. Ils en concluaient que cette technologie pouvait être utilisée, tant pour l'enrichissement spécifique en caséine native des laits de fromagerie, que pour la préparation à l'état purifié d'un nouveau produit : le phosphocaséinate natif ou PPCN.

Dans la présente étude, nous nous sommes attachés à préciser les conditions optimales de mise en œuvre de la technologie de microfiltration sur membrane, avec les équipements dont nous disposions et en prenant en compte les observations réalisées, notamment en matière d'épuration bactérienne (Trouvé et al, 1991). Notre objectif a été également de caractériser analytiquement le fractionnement du lait ainsi réalisé et de déterminer les propriétés de solubilité et de coagulabilité par la présure du PPCN.

\section{MATÉRIEL ET MÉTHODES}

\section{Lait utilisé}

Le lait utilisé était un lait de grand mélange écrémé et cru, en provenance de la laiterie Préval (Montauban-de-Bretagne, France), épuré par microfiltration sur membrane ayant un diamètre de pores de 1,4 $\mu \mathrm{m}$, comme décrit par Trouvé et al (1991); 180 I de lait étaient mis en œuvre à chaque expérimentation.

\section{Séparation du phosphocaséinate (PPCN) par microfiltration}

L'installation utilisée était celle décrite par Trouvé et al (1991) mais les membranes mises en œuvre avaient un diamètre de pores de $0,2 \mu \mathrm{m}$ (SCT, Tarbes, France). La température de fonctionnement était maintenue à $50^{\circ} \mathrm{C}$. Le rétentat quittant la boucle de recirculation au débit de $500 \mathrm{l}^{\circ} \mathrm{h}^{-1} \bullet \mathrm{m}^{-2}$ était recirculé dans le bac d'alimentation. La vitesse de balayage de la membrane du côté du rétentat était de l'ordre de

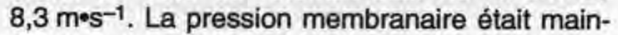
tenue entre 0,6 et 0,9 bar, selon les essais. Le débit de perméation moyen était de $801 \cdot h^{-1} \cdot \mathrm{m}^{-2}$ jusqu'à obtention d'un facteur de concentration volumique $(F C V)$ de 3 . La concentration du réten- 
tat a été estimée en calculant le facteur de concentration volumique $(F C V): F C V=V R N L$; $V R$, volume initial de lait, $V L$, volume de rétentat). La diafiltration du rétentat était alors réalisée par addition en continu de 240 I d'eau déminéralisée, ce qui correspond à un taux de diafiltration de 4 ( $\alpha=$ volume d'eau / volume de rétentat). Le nettoyage des membranes était réalisé comme indiqué par Trouvé et al (1991). La résistance hydraulique de la membrane de microfiltration était calculée selon la formule suivante :

$$
R_{\mathrm{cl}}=\left(P T M / J \times \mu_{\mathrm{p}}\right)-R_{\mathrm{m}}
$$

PTM étant la pression transmembranaire moyenne, $J$ le flux de perméation, $\mu_{\mathrm{p}}$ la viscosité du microfiltrat, $R_{m}$ la résistance hydraulique de la membrane propre.

\section{Concentration du PPCN par ultrafiltration}

Le rétentat de microfiltration diafiltré était concentré par ultrafiltration à l'aide de l'équipement Techsep (Miribel, France) décrit par Goudédranche et al (1980).

\section{Séchage du PPCN}

II était réalisé par atomisation dans une tour pilote Niro (Nanterre, France) ayant une capacité évaporatoire de $15 \mathrm{~kg}$ d'eau/h. Les températures d'entrée et de sortie de l'air étaient respectivement de $175 \pm 1^{\circ} \mathrm{C}$. L'atomisation était réalisée à l'aide d'une turbine tournant à $22800 \mathrm{t} /$ min.

\section{Déterminations analytiques}

La composition chimique des échantillons a été déterminée en utilisant les méthodes d'analyse suivantes : extrait sec total (EST), $7 \mathrm{~h}$ à l'étuve à $105^{\circ} \mathrm{C}$; cendres, $5 \mathrm{~h}$ au four à $550^{\circ} \mathrm{C}$; lactose : méthode de Acton (1977); turbidité : densité optique à $600 \mathrm{~nm}$; matières azotées totales (MAT) : $6,38 \times$ azote dosé par la méthode de Kjeldahl; fractionnement de la MAT : azote non caséique (NCN), azote non protéique (NPN) selon Rowland (1938); minéraux ( $\mathrm{Ca}, \mathrm{Mg}, \mathrm{Na}$, K) par spectrophotométrie d'absorption atomique; phosphore, dosage colorimétrique après minéralisation selon la norme FIL (1982).

Dosage des protéines par HPLC : appareil Varian 5000 (Varian, Les Ulis, France), colonne C18 thermostatée à $40^{\circ} \mathrm{C}$. Séparation par gradient d'acétronitrile : tampon A : eau TFA $0,1 \%$; tampon B : eau (20) acétronitrile (80) TFA $0,1 \%$. Débit $1 \mathrm{ml} / \mathrm{min}$. Pour le dosage des protéines $\alpha$ lactalbumine $(\alpha-\mathrm{La})$ et $\beta$-lactoglobuline $(\beta-\mathrm{Lg})$, on utilisait : une colonne Nucléosil C18 3005 $\mu \mathrm{m}$ de $25 \mathrm{~cm}$ avec un gradient d'élution de 44 à $62 \%$ de tampon $B$ en 30 min et une détection à $280 \mathrm{~nm}$. Pour le dosage des protéines dans les produits diafiltrés, on utilisait : une colonne Vydac 218 TP5 $5 \mu \mathrm{m}$ de $25 \mathrm{~cm}$ avec un gradient de 35 à $65 \%$ de tampon $B$ en $60 \mathrm{~min}$ et une détection à $215 \mathrm{~nm}$. Le dosage d' $\alpha$-La et de $\beta$ - $\mathrm{Lg}$ de la poudre a également été fait par immunodiffusion en utilisant la technique de Ingild (1983). Les électrophorèses ont été réalisées, soit en milieu SDS selon Laemmli (1970), soit en appliquant la méthode décrite par Andrews (1983). Pour la mesure des diamètres micellaires, un compteur de particules N4MD Coultronics (Margency, France) a été utilisé. La détermination a été effectuée sur une solution de PPCN à $30 \mathrm{~g}^{\circ \mathrm{kg}^{-1}}$. L'extraction des phospholipides de la poudre à été réalisée selon la méthode de Folch et al (1957), modifiée par Bligh et Dyer (1959) et leur séparation par chromatographie en couche mince (Mangold et Kammereck, 1962) avec une détection à l'iode. La coagulation par la présure (présure commerciale $\mathrm{F}$ $1 / 8000$ ) a été étudiée à l'aide d'un Formagraph (Foss Electric, Nanterre, France) à la température de $32{ }^{\circ} \mathrm{C}$. Sur l'enregistrement, les paramètres suivants ont été relevés : temps de coagulation (TC); vitesse de raffermissement $K_{20}=$ temps pour obtenir un écartement de $2 \mathrm{~cm}$ ) et fermeté ( $E_{30}$, écartement après $30 \mathrm{~min}$ ). La solubilité des poudres a été estimée selon la méthode normalisée de la FIL (1988).

\section{RÉSULTATS}

\section{Performances de perméation}

Un essai de microfiltration (essai 1), conduit jusqu'à des teneurs en MAT éle- 
vées $\left(F C V 6 ; M A T=160 \mathrm{~g}^{\circ} \mathrm{kg}^{-1}\right)$, a permis d'étudier la variation du flux de perméation (J), au cours de la concentration. La PTM a été maintenue entre 0,6 et 0,7 bar pendant toute la durée de l'expérimentation, soit $2 \mathrm{~h}$ environ, en diminuant la pression dans la boucle de perméat, ce qui se traduisait par une réduction des flux de 70 à $20 l \bullet h^{-1} \bullet \mathrm{m}^{-2}$. La figure 1 montre que le produit $F C V \times J$ passe par un maximum pour une teneur en MAT du rétentat de $120 \mathrm{~g} \mathrm{~kg}^{-1}$.

Pour la préparation du PPCN, la microfiltration a été conduite jusqu'à un FCV de l'ordre de 3 , soit à une teneur en protéines de $80 \mathrm{~g}^{\circ} \mathrm{kg}^{-1}$ environ. La figure 2 montre l'évolution de la PTM et des flux lors de 2 essais successifs. Lors de l'essai 2, la PTM a été maintenue à 0,8 bar environ pendant la concentration, avec un flux variant de 60 à $30 l \bullet h^{-1} \bullet \mathrm{m}^{-2}$ (fig 2). Pendant la diafiltration, il a été possible d'augmenter la PTM jusqu'à 1,4 bar sans observer de colmatage et par là-même, d'obtenir un débit de perméation de $70 l \cdot h^{-1} \bullet \mathrm{m}^{-2}$. Lors de l'essai 3, la PTM n'a pu être maintenue et est montée jusqu'à 1,3 bar. La résistance hydraulique correspondant au colmatage de la membrane $\left(R_{\mathrm{f}}\right)$ a été calculée; elle variait de $3 \cdot 10^{12}$ à $2110^{12} \mathrm{~m}^{-1}$ dans l'essai 1 lorsque le FCV augmentait

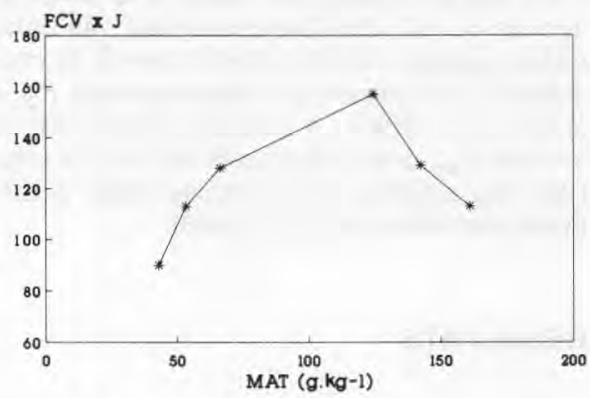

Fig 1. Évolution des conditions de perméation au cours de la concentration du lait par microfiltration (essai 1).

Permeation performances during milk concentration by microfiltration. de 1 à 6 (fig 3). Au cours de la diafiltration, elle présentait une valeur moyenne de $11 \cdot 10^{12} \mathrm{~m}^{-1}$.

\section{Fractionnement du lait}

L'analyse des laits, rétentats $(R)$ et perméats $(P)$ prélevés au cours des expérimentations a permis de suivre le fractionnement opéré par la membrane. Le tableau I présente la composition chimique des produits initiaux et finaux prélevés lors d'une expérience de microfiltration. L'évolution de la composition des perméats et des rétentats au cours d'un essai est présentée graphiquement sur les figures 4 et 5. Les perméats obtenus lors de la
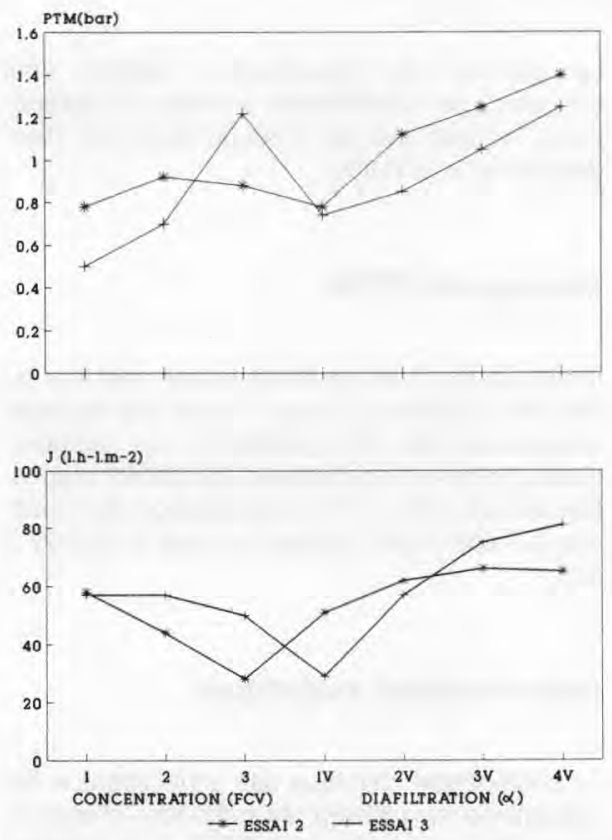

Fig 2. Évolution de la pression transmembranaire moyenne et du flux au cours de la microfiltration.

Evolution of mean transmembrane pressure and fluxes. 


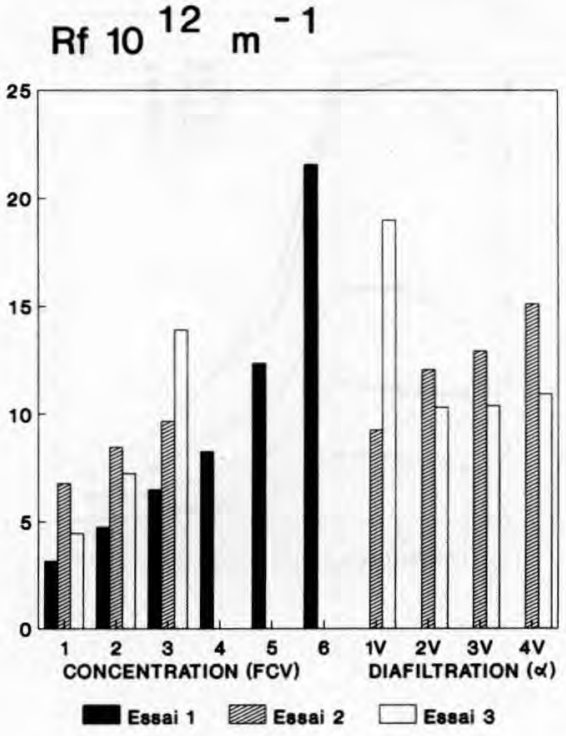

Fig 3. Résistance hydraulique de la membrane de microfiltration.

Hydraulic resistance in the microfiltration system. concentration par microfiltration sont parfaitement limpides. Ils ont une teneur en matière sèche de l'ordre de $59 \mathrm{~g}^{\circ} \mathrm{kg}^{-1}$ dont le constituant principal est le lactose (45 $\left.\mathrm{g} \mathrm{kg}^{-1}\right)$. La fraction minérale est présente à des teneurs correspondant à la fraction minérale non colloïdale du lait. La MAT des perméats $\left(6,3 \mathrm{~g} \mathrm{~kg}^{-1}\right)$ contient, outre une fraction $N P N$ égale à celle du lait $(1,9$ $\left.\mathrm{g}^{\circ} \mathrm{kg}^{-1}\right)$, des protéines solubles, I' $\alpha$-La $(0,8$ $\mathrm{g}^{\circ} \mathrm{kg}^{-1}$ ) et la $\beta-\mathrm{Lg}\left(3,0 \mathrm{~g} \bullet \mathrm{kg}^{-1}\right)$. La présence de caséine (fraction précipitable à pH 4,6 = $0,4 \mathrm{~g}^{\circ} \mathrm{kg}^{-1}$ ) est révélée également sur les électrophorèses de la figure 6. La composition du perméat évolue peu au cours de la phase de concentration; on remarque cependant que le perméat initial est légèrement dilué, ceci est certainement dû à l'eau restant dans les circuits de l'installation.

Les rétentats ont une teneur en MAT qui augmente au cours de la concentration (fig 5) jusqu'à atteindre une teneur de l'ordre de $75,6 \mathrm{~g}^{\circ} \mathrm{kg}^{-1}$. Le constituant principal en est la caséine dont la teneur aug-

Tableau I. Composition des produits obtenus lors de la microfiltration. Composition of products obtained during microfiltration.

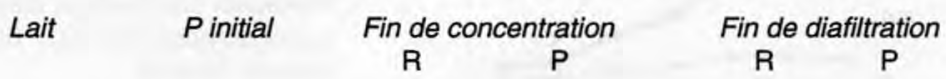

\begin{tabular}{|c|c|c|c|c|c|c|}
\hline $\mathrm{DO} \times 10^{3}$ & & 6 & & 9 & & 2 \\
\hline $\mathrm{pH}\left(\mathrm{g}^{\circ} \mathrm{kg}^{-1}\right)$ & 6,6 & 6,5 & 6,6 & 6,5 & 6,9 & 6,8 \\
\hline EST & 88,4 & 52,0 & 131,9 & 59,3 & 86,6 & 2,1 \\
\hline$M A T$ & 32,2 & 5,7 & 75,6 & 6,3 & 76,4 & 0,6 \\
\hline $\operatorname{NCN}(\times 6,38)$ & 7,6 & 5,2 & 9,8 & 5,9 & 4,8 & 0,6 \\
\hline$N P N(\times 6,38)$ & 2,1 & 1,7 & 1,8 & 1,9 & 0,4 & 0,3 \\
\hline$\beta$-Lactoglobuline & 3,1 & 2,7 & 3,0 & 3,0 & 0,4 & 0,3 \\
\hline 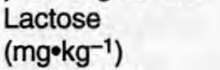 & 45,0 & 40,0 & 41,0 & 45,0 & 1,0 & 1,3 \\
\hline $\mathrm{P}$ & 989 & 359 & 1925 & 405 & 1649 & 25 \\
\hline $\mathrm{Ca}$ & 1232 & 306 & 2719 & 329 & 2691 & 38 \\
\hline $\mathrm{Mg}$ & 109 & 64 & 159 & 72 & 104 & 6 \\
\hline $\mathrm{K}$ & 1511 & 1251 & 1505 & 1436 & 77 & 43 \\
\hline $\mathrm{Na}$ & 414 & 341 & 409 & 380 & 28 & 24 \\
\hline
\end{tabular}



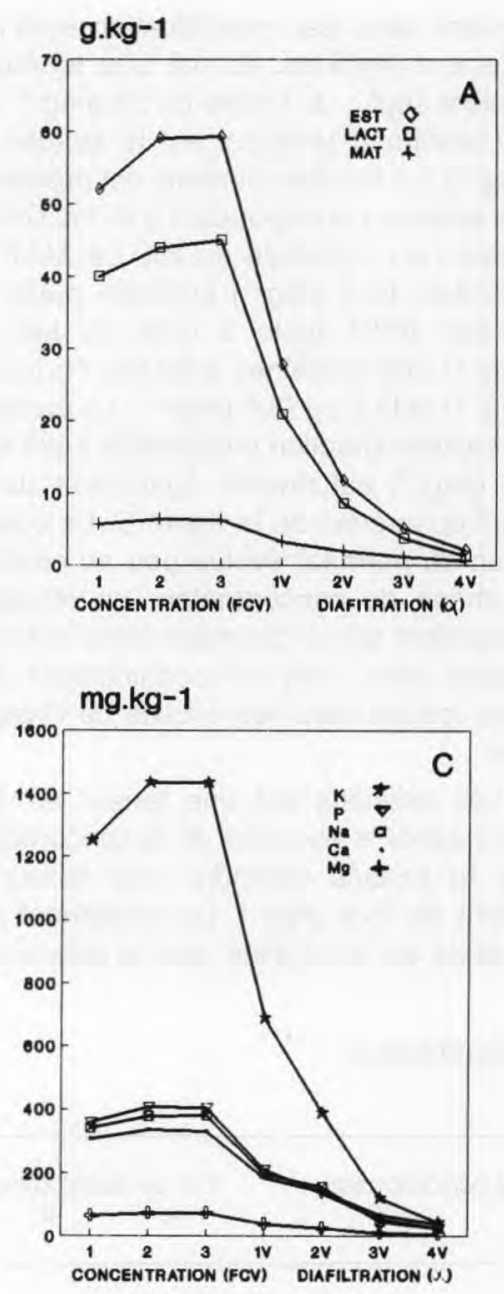

mente de 24,6 à $69,8 \mathrm{~g}^{\circ} \mathrm{kg}^{-1}$ (MAT NCM). Le facteur de concentration de la caséine est donc de $69,8 / 24,6=2,84$. La fraction azotée du rétentat non précipitable à $\mathrm{pH} 4,6$ (NCN) augmente également de 7,6 à $9,8 \mathrm{~g}^{\circ} \mathrm{kg}^{-1}$, bien que ses constituants principaux, NPN $\left(1,9 \mathrm{~g}^{\circ} \mathrm{kg}^{-1}\right)$ et $\alpha-\mathrm{La}+\beta$ $\mathrm{Lg}\left(0,8 \mathrm{~g}^{\circ} \mathrm{kg}^{-1}+3,0 \mathrm{~g} \bullet \mathrm{kg}^{-1}\right)$ y aient une teneur constante. Certains constituants du NCN du lait sont donc retenus par la membrane. Les profils électrophorétiques (fig 6) mettent en évidence dans les réten-

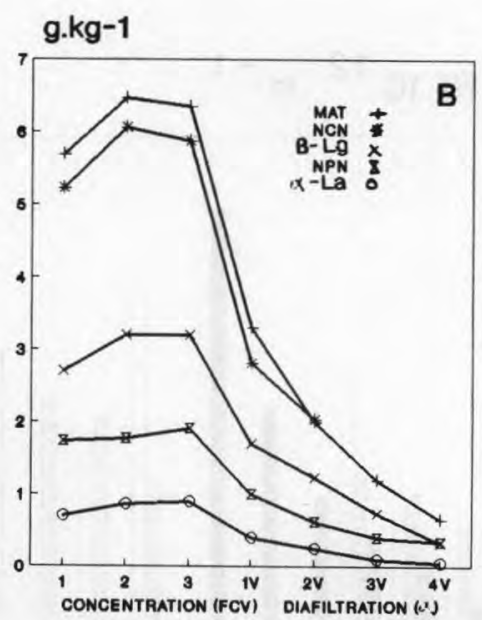

Fig 4. Évolution de la composition des perméats au cours de la microfiltration. (A) : Composition globale; (B) : fraction azotée; (C) : minéraux. Permeate composition during microfiltration $(A)$ : gross composition; $(B)$ : nitrogen fractions; $(C)$ : mineral content.

tats, outre les caséines, des constituants de poids moléculaire $>60000$ Da fortement retenus par la membrane, parmi lesquels figurent probablement la sérum albumine bovine, les immunoglobulines et les phospholipoprotéines. Une analyse qualitative par chromatographie en couche mince a d'ailleurs confirmé la présence de toutes les classes de phospholipides du lait dans le PPCN. Les électrophorèses révèlent également dans les rétentats des constituants peptidiques de poids molécu- 


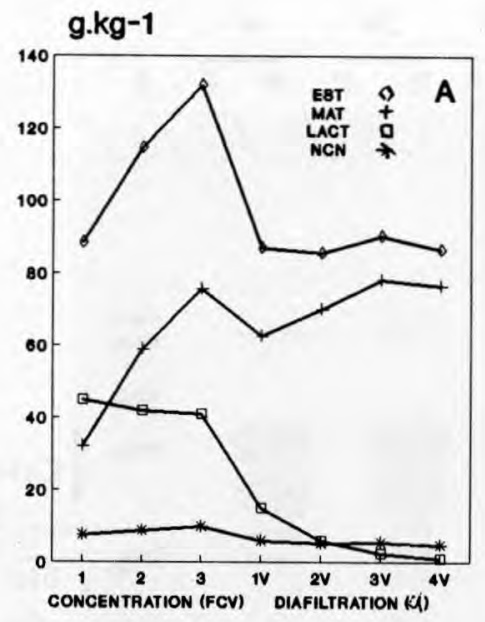

mg.kg-1

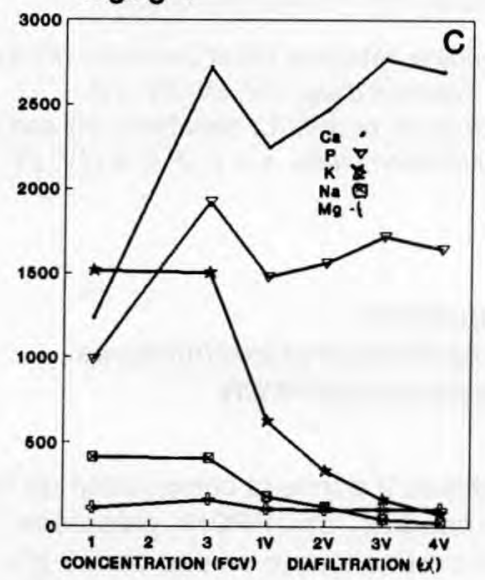

laires apparents 10000 et $20000 \mathrm{Da}$ retenus par la membrane. La fraction 20000 $\mathrm{Da}$ est présente dans le profil électrophorétique du NCN des rétentats (résultats non présentés), et donc soluble à pH 4,6.

La diafiltration permet l'élimination des solutés du rétentat capables de traverser la membrane (figs 4,5 ). L'élimination des constituants micromoléculaires en solution dans le rétentat s'effectue selon une décroissance logarithmique, en ce qui concerne le lactose (fig 7 ) : le rapport $\mathrm{Ln}$

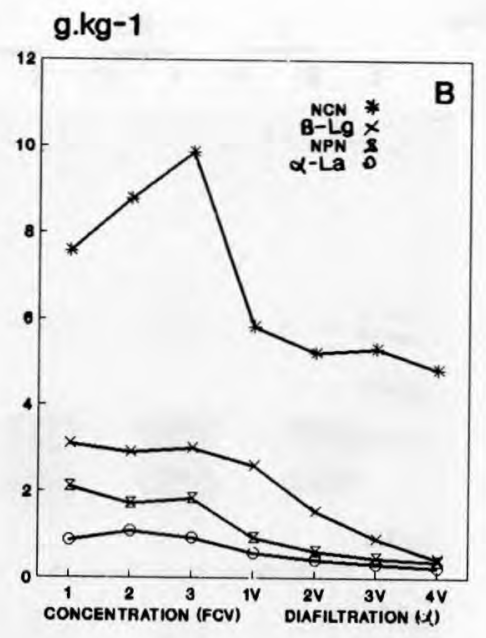

Fig 5. Évolution de la composition des rétentats au cours de la microfiltration. (A) : composition globale; $(B)$ : fractions azotées; $(C)$ : minéraux. Retentate composition during microfiltration $(A)$ : Gross composition; $(B)$ : nitrogen fractions; $(C)$ : mineral content.

$C / C_{0}$ décroît en fonction du taux de diafiltration $(C=$ concentration du soluté au taux de diafiltration étudié, $C_{0}$, sa concentration initiale dans le rétentat). Pour le potassium et le sodium, on observe une faible rétention apparente qui est due pour la plus grande partie à l'apport de ces minéraux dans l'eau de diafiltration ( $\mathrm{K}=$ $8 \mathrm{mg}^{\circ} \mathrm{kg}^{-1}, \mathrm{Na}=29 \mathrm{mg}^{\circ} \mathrm{kg}^{-1}$ ). Les minéraux liés aux protéines : phosphore, calcium et magnésium sont retenus. Le perméat final ne contient plus que $2,1 \mathrm{~g}^{\circ} \mathrm{kg}^{-1}$ 


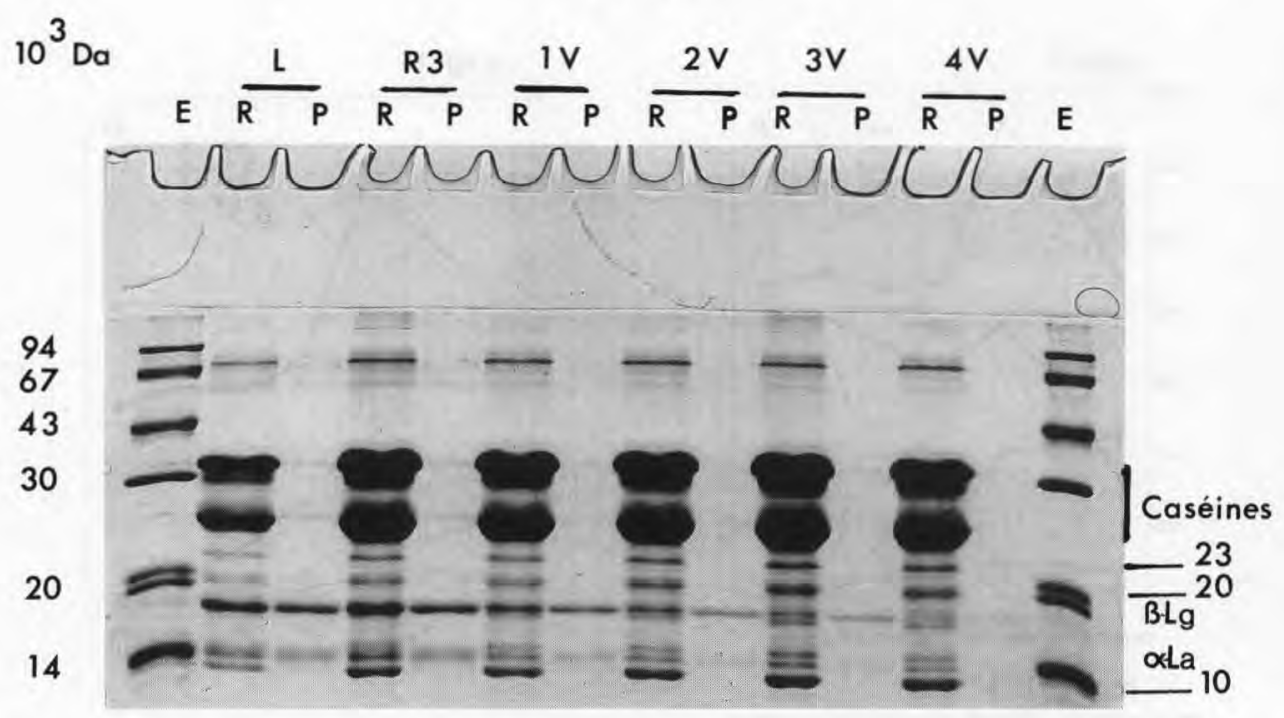

Fig 6. Electrophorèse en SDS : $E$, étalons de poids moléculaire; rétentats $(R)$ et perméats $(P)$ du lait $(L)$; rétentat 3 non diafiltré $\left(R_{3}\right)$, puis diafiltré avec 1, 2, 3, 4 volumes d'eau (1V, 2V, 3V, $4 \mathrm{~V}$ ).

Electrophoresis (SDS): (E) Molecular weight references; analysis of milk (L) retentates $(\mathrm{R})$ and permeates $(\mathrm{P})$ at the end of concentration $\left(\mathrm{R}_{3}\right)$ and after 4 diafiltration steps, $\alpha=1,2,3,4(1 \mathrm{~V}, 2 \mathrm{~V}, 3 \mathrm{~V}$, $4 V)$.

d'extrait sec, ce qui représente un taux de dilution de la phase aqueuse d'environ 30 . La $\beta$ - Lg est éliminée moins rapidement que les minéraux.

\section{Évolution de la purification du PPCN en fonction du taux de diafiltration}

La figure 8 montre le suivi de la purification de la MAT du rétentat au cours d'un essai. Le rapport MAT/EST augmente au cours de la microfiltration, de $36 \%$ dans le lait à $61 \%$ à la fin de la concentration. Au cours de la diafiltration, ce rapport devient égal à $72,82,86,88 \%$ pour des taux de diafiltration de $1,2,3,4$. Les protéines solubles microfiltrables sont éliminées pendant la diafiltration, comme le montre l'évolution de la teneur en $\beta$ - Lg. Le rapport $\beta$ $\mathrm{Lg} / E S T$ passe de 2,2 à $0,2 \%$ au cours de la diafiltration.

\section{Composition et propriétés physicochimiques de la poudre de PPCN}

Le tableau II donne la composition de l'une des poudres de PPCN préparées. La poudre a une teneur en eau de $5,9 \mathrm{~g} \%$. Sa matière sèche contient $88,9 \mathrm{~g} \%$ de MAT et $9,0 \mathrm{~g} \%$ de cendres. La teneur en minéraux solubles est très faible par rapport aux minéraux totaux. La caséine est sous forme de micelles et la répartition des tailles micellaires est analogue à celle d'un lait cru (tableau III). Le diamètre moyen des micelles est de $258 \mathrm{~nm}$ dans la solution de PPCN, et de $235 \mathrm{~nm}$ pour le lait cru. La dispersion des tailles autour de la moyenne est également comparable : $80 \%$ des micelles entre 183 et $367 \mathrm{~nm}$ pour le PPCN et entre 165 et $335 \mathrm{~nm}$ pour le lait cru. La solubilité des poudres a été déterminée sur des solutions à $10 \%$. Le taux 


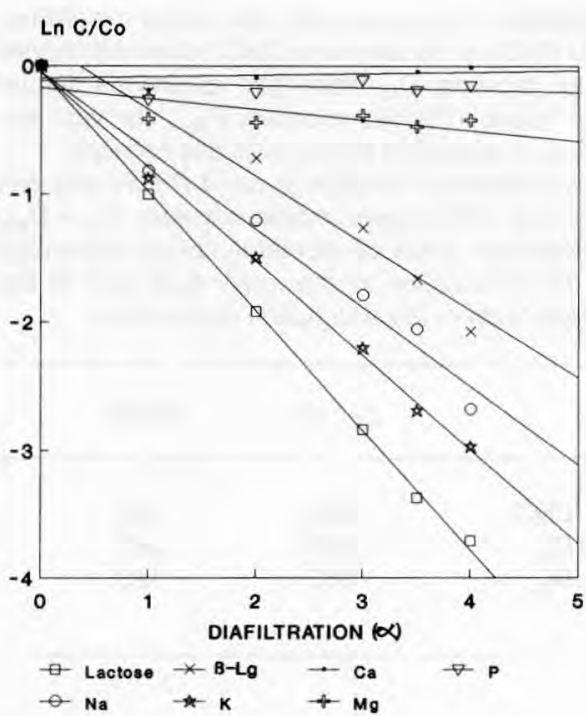

Fig 7. Élimination des solutés au cours de la diafiltration des rétentats.

Solute elimination in retentates during diafiltration.

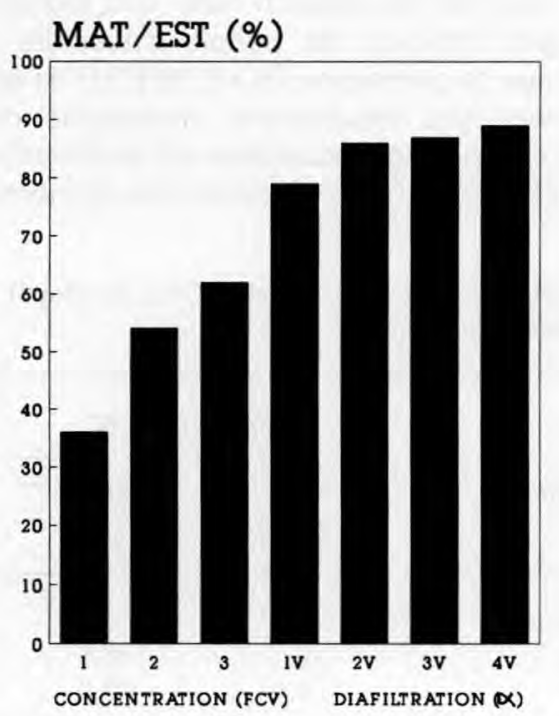

d'insoluble obtenu à $50^{\circ} \mathrm{C}$ est de $2,8 \%$ (v/v); à $20^{\circ} \mathrm{C}$, il est de $26 \%$. Cependant, si la solution obtenue à $50^{\circ} \mathrm{C}$ est réfroidie à $20^{\circ} \mathrm{C}$, le même taux d'insoluble $(2,8 \%)$ est observé à cette température.

Nous avons étudié l'aptitude à la coagulation par la présure de la solution de PPCN. Le tableau IV permet de comparer la coagulation d'une suspension du PPCN reconstitué à $30 \mathrm{~g}^{\circ} \mathrm{kg}^{-1}$ et celle d'un lait cru (teneur en caséine du PPCN et du lait : $25 \mathrm{~g} \mathrm{~kg}^{-1} ; \mathrm{pH} \mathrm{6,62)}$. Le temps de coagulation (TC) du PPCN (6,5 min) est environ 2 fois plus court que celui du lait cru (14 min). Après un traitement thermique à $100^{\circ} \mathrm{C}-5$ min, le TC du PPCN est de 7,8 min, il augmente donc de $20 \%$ par rapport au produit non chauffé. Le lait chauffé dans les mêmes conditions ne présente aucune coagulation à $40 \mathrm{~min}$. Même après le traitement thermique, le TC du PPCN (7,8 min) reste encore inférieur à celui du lait cru (14 min). De plus, la vitesse du raffermissement du

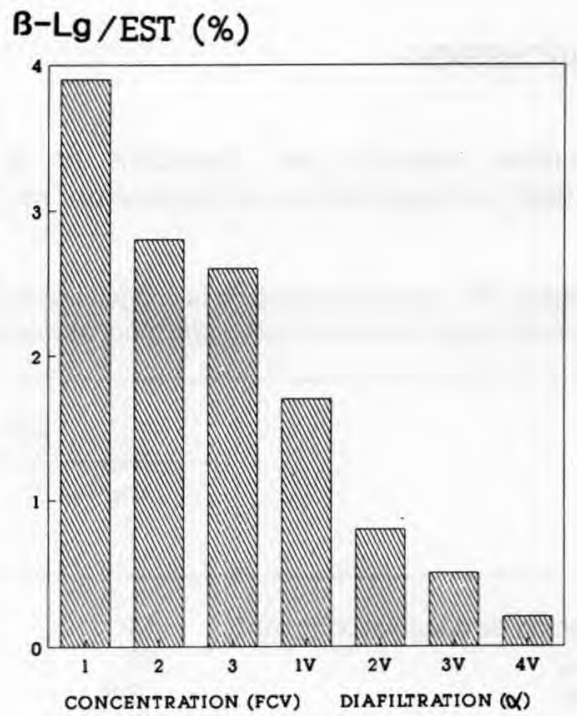

Fig 8. Purification du phosphocaséinate natif au cours de la diafiltration. Native phosphocaseinate purification during the diafiltration process. 
Tableau II. Composition chimique d'une poudre de PPCN (\% de matière sèche).

Chemical composition of a PPCN powder (\% dry matter).

\begin{tabular}{lcc}
\hline Matière azotée totale, dont & & 88,9 \\
NCN & & 6,3 \\
NPN & & 0,6 \\
B-lactoglobuline & & 0,4 \\
Lactose & & 1,0 \\
Cendres & & 0,9 \\
Minéraux & & \\
Calcium & Totaux & Solubles \\
Potassium & 3,04 & 0,06 \\
Sodium & 0,06 & 0,02 \\
Phosphore & 0,13 & 0,01 \\
& 1,95 & - \\
\hline
\end{tabular}

caillé de PPCN $\left(K_{20}\right)$ est beaucoup plus rapide que celle du lait pour les produits crus (10 min au lieu de $23 \mathrm{~min}$ ). Le traitement thermique la ralentit $(16,5 \mathrm{~min})$ mais elle reste supérieure à celle du lait cru.

\section{DISCUSSION}

Comme supposé par Fauquant et al (1988), le traitement du lait écrémé par mi-
Tableau III. Comparaison des tailles micellaires du PPCN et du lait $(\mathrm{nm})$. DMO: diamètre moyen des micelles; $D_{10}$ : diamètre au-dessus duquel on trouve $10 \%$ des micelles; $D_{90}$ : diamètre audessus duquel on trouve $90 \%$ des micelles.

Comparison of micellar sizes of PPCN and milk (in $\mathrm{nm}$ ). DMO: mean micelle diameter; $\mathrm{D}_{10}-\mathrm{D}_{90}$ : dispersion limits of diameter values discording $10 \%$ of micelles in the lower side and in the upper side of the distribution respectively.

\begin{tabular}{lll}
\hline & Lait cru & PPCN \\
\hline & & \\
$D M O$ & 235 & 258 \\
$D_{10}$ & 335 & 367 \\
$D_{90}$ & 165 & 183 \\
& & \\
\hline
\end{tabular}

crofiltration sur membrane ayant un diamètre de pores voisin de $0,2 \mu \mathrm{m}$, traitement combiné avec une diafiltration, permet la séparation et la purification de la caséine micellaire dans l'état où elle se trouve dans le lait, c'est-à-dire du phosphocaséinate de calcium natif. Les technologies utilisées ont permis d'atteindre un taux de purification ( $\mathrm{N} \times 6,38 / E S T)$ de $89 \%$ avec des équipements modulaires dont l'extrapolation industrielle est aisément envisageable, sous réserves d'un ajustement

Tableau IV. Caractéristiques de la coagulation du PPCN par la présure (solution PPCN à $30 \mathrm{~g}^{\circ} \mathrm{kg}^{-1}$ ). Rennet coagulation of a native phosphocaseinate solution $\left(30 \mathrm{~g}^{\circ} \mathrm{kg}^{-1}\right)$.

\begin{tabular}{|c|c|c|c|c|}
\hline & \multicolumn{2}{|c|}{ Lait } & \multicolumn{2}{|c|}{ Solution PPCN } \\
\hline & Cru & Chauffé * & $\begin{array}{l}\text { Sans } \\
\text { traitement }\end{array}$ & Chauffée * \\
\hline Temps de coagulation (min) & 14 & $>40$ & 6,5 & 7,8 \\
\hline$K_{20}$ & 23 & - & 10 & 16,5 \\
\hline$E_{30}$ & 2,8 & - & 4,4 & 3,0 \\
\hline
\end{tabular}

* Traitement thermique à $100^{\circ} \mathrm{C}-5$ min suivi d'un ajustement du pH à 6,62.

Heat treatment $\left(100^{\circ} \mathrm{C}-5 \mathrm{~min}\right)$ followed by $\mathrm{pH}$ adjustment at 6.62 . 
des paramètres hydrauliques à l'évolution des caractéristiques rhéologiques du rétentat de microfiltration.

Avec l'équipement de microfiltration utilisé, conçu sur le plan hydraulique pour la recirculation d'un rétentat ne présentant pas d'évolution notable de sa viscosité, les résultats rassemblés dans la figure 1 montrent que le FCV optimal de la phase de concentration serait de 4,2 , ce qui correspond à une teneur en MAT de $124,4 \mathrm{~g}^{\circ} \mathrm{kg}^{-1}$ et à un rapport caséine/MAT de $91 \%$. Au-delà de ce $F C V$, la résistance hydraulique de la membrane consécutive à l'accroissement du colmatage total s'élève brutalement (fig 3 ), ce qui conduit à une chute inacceptable des flux de perméation et à une rétention élevée des protéines solubles. Une grande variabilité des performances de filtration a été observée. Les différences constatées sur les flux initiaux, toutes conditions de mise en œuvre étant égales par ailleurs, $80 \mathrm{l}^{\circ} \mathrm{h}^{-1} \bullet \mathrm{m}^{-2}$ (résultats non présentés) à $60 \mathrm{l}^{-1} \mathrm{~h}^{-1} \cdot \mathrm{m}^{-2}$ (fig 2) conduisent à supposer que le protocole de nettoyage utilisé n'était pas pleinement efficace et que la propreté chimique des membranes n'était pas fidèlement retrouvée. La présence de PPCN (jusqu'à $3 \mathrm{~g}^{\bullet} \mathrm{kg}^{-1}$ ) et de protéines solubles de haut poids moléculaire dans les premières fractions de microfiltrat collectées, lors d'essais à flux initiaux de perméation élevés, conduit à penser qu'une optimisation est à réaliser, soit au niveau des membranes (diamètre des pores; distribution de la taille de ces pores; nature du matériau...), soit au niveau de la mise en régime de l'équipement. La définition de conditions optimales de nettoyage et de mise en régime devrait également réduire les variations de flux de perméation observées d'un essai à l'autre, lors des phases de concentration (fig 2).

L'opération de diafiltration a été réalisée, par précautions, à FCV 2,7 (MAT $=75,6$ $\mathrm{g}^{\circ} \mathrm{kg}^{-1}$ ) en raison des caractéristiques de l'équipement de microfiltration utilisé. Du choix de ce FCV ont évidemment résulté un allongement de la durée de la phase de diafiltration et une augmentation de la quantité de liquide de diafiltration ajouté. L'expression mathématique de la diafiltration continue peut, en effet, s'écrire sous la forme:

$T_{\mathrm{D}}$ (temps de diafiltration) $=\frac{V L}{F C V \times J} \operatorname{Ln} C_{\mathrm{d}} / C$

avec $C_{0}$, concentration initiale des microsolutés dans le rétentat et $C$, concentration des microsolutés dans le rétentat diafiltré (Peri et al, 1973; Kiviniemi, 1977). Pour une diafiltration donnée, $T_{D}$ passe par un minimum quand la valeur du produit $F C V \times J$ atteint son maximum, soit dans les conditions de cette étude quand la teneur en MAT du rétentat est de $120 \mathrm{~g}^{\circ} \mathrm{kg}^{-1}$ (FCV 4,2).

L'ajout de 4 diavolumes d'eau au rétentat de microfiltration a permis d'éliminer $96 \%$ des constituants micromoléculaires de ce produit, calcul effectué à partir des teneurs en substance sèche des perméats (tableau I). La teneur en lactose $(77 \%$ de la substance sèche microfiltrable) a été réduite de près de $98 \%$ par le cumul des phases de concentration et de diafiltration. Ces résultats sont en bon accord avec les valeurs prédéterminées à l'aide des lois de la diafiltration (Peri et al, 1973; Kiviniemi, 1977), que ce soit sur la base du lactose ou sur la base de la substance sèche initiale microfiltrable, (ES) p (tableau I). La relation entre le degré de purification du PPCN (caséine + minéraux colloïdaux) et le nombre $(\alpha)$ de diavolumes d'eau utilisés dans la phase de diafiltration découle, en effet, de la relation:

$$
\frac{P P C N}{E S T}=\frac{P P C N}{P P C N+(E S)_{p} \mathrm{e}^{-\alpha}}
$$

L'utilisation de fluides de diafiltration autres que l'eau est envisageable. Toute 
une gamme de nouveaux produits pourrait ainsi être proposée. Par exemple, l'utilisation d'ultrafiltrat de lait conduira à un enrichissement en PPCN du rétentat mais sans autre modification de la phase soluble que l'abaissement de la teneur en protéines de lactosérum. L'emploi de solutions diluées d'acides ou de complexants de cations divalents est à même de permettre l'obtention de caséine micellaire partiellement déminéralisée. À l'inverse, la diafiltration avec des solutions minérales peut conduire à un enrichissement en cations présentant soit un intérêt technologique, soit un intérêt nutritionnel.

Contrairement à ce qui pouvait être attendu, une rétention élevée de peptides ayant un poids moléculaire apparent de l'ordre de 10000 à $20000 \mathrm{Da}$ a été observée avec la membrane de microfiltration utilisée (fig 6). Ces constituants identifiables aux protéoses-peptones (Mati et al, 1991) se trouvent donc dans le lait à l'état d'agrégats de haut poids moléculaire retenus à peu près totalement par la membrane.

Leur présence dans le produit final (poudre de PPCN) ne diminue que très légèrement la "pureté caséique» de cette poudre puisque plus des $2 / 3$ des protéoses-peptones sont constitués par des fragments de la caséine $\beta$ (Mati et al, 1991).

La quantité de protéines solubles de haut poids moléculaire retenue dans le rétentat diafiltré et donc dans la poudre de PPCN, dépend, comme nous l'avons dit plus haut, des conditions de perméation de l'équipement de microfiltration. Mais, que ce soit pour ce groupe de protéines ou pour la $\beta-\mathrm{Lg}$, la proportion présente dépendra aussi de l'intensité des traitements thermiques appliqués lors de l'écrémage et de la durée des phases de microfiltration et de diafiltration (rapports entre les surfaces de membrane et les volumes morts).
II résulte des rétentions protéiques observées que le microfiltrat obtenu dans la phase de microfiltration est un produit relativement simple sur le plan protéique. Contenant principalement de la $\beta$ - Lg et de l' $\alpha$-La, il constitue une matière première de choix pour la séparation de ces deux protéines par les méthodes de précipitation différentielle proposées par Pearce (1983) et par Pierre et Fauquant (1986) ou par ultrafiltrations en cascade (Roger et al, 1979). Chacune de ces deux protéines devrait pouvoir être obtenue avec un taux de pureté élevé, compte tenu de l'absence dans le microfiltrat de peptides dérivés des caséines $\beta$ et $\kappa$ et des phospholipoprotéines.

La poudre de PPCN préparée présentait une solubilité à $50^{\circ} \mathrm{C}$ satisfaisante $(97,2 \% \mathrm{v} / \mathrm{v})$ mais faible à $20^{\circ} \mathrm{C}(74 \% \mathrm{v} / \mathrm{v})$. Ce comportement de solubilité est caractéristique d'une déshydratation trop rapide du produit injecté en tour d'atomisation (Webb et al, 1978). L'abaissement du taux d'insoluble à $20^{\circ} \mathrm{C}$ devrait résulter d'une adaptation entre les caractéristiques physico-chimiques du rétentat et les températures d'entrée et de sortie de tour accompagnée éventuellement d'une stratification du processus de déshydratation : utilisation de 1 ou 2 lits fluidisés.

Le degré de "pureté caséique» de la poudre obtenue peut être estimé à partir de la composition du tableau II. Les matières azotées précipitables à $\mathrm{pH} 4,6$ représentent $82,6 \%$ de la substance sèche. La caséine étant sous forme micellaire, une proportion de sels phosphocalciques, égale à $8,5 \%$ du produit $N \times 6,38$, fait partie intégrante de sa structure (Farrell, 1988). De plus, à partir de la composition moyenne des protéines de lactosérum (Alais, 1984) et des observations réalisées sur les taux de perméation de ces protéines (tableau I), il peut être estimé qu'environ $40 \%$ des matières azotées de la poudre non précipitables à $\mathrm{pH} 4,6$ 
sont des fragments de caséine (protéosespeptones). La minéralisation de ces protéoses-peptones étant mal connue, elle ne peut être prise en compte, il résulte de cet ensemble de constatations que la teneur en matériau caséique (caséine micellaires + protéoses-peptones) représente $92,1 \%$ de la substance sèche de la poudre.

La meilleure aptitude à la transformation fromagère du PPCN par rapport au lait cru, appréciée par une réduction de $53 \%$ du temps de coagulation par la présure et une accélération de $56 \%$ de l'organisation du gel $\left(K_{20}\right.$ et $\left.E_{30}\right)$ découle de l'élimination par microfiltration-diafiltration des constituants de la phase soluble du lait exerçant un effet défavorable sur la coagulation enzymatique du lait (Dalgleish, 1982). L'abaissement des teneurs en lactose et en sels minéraux solubles facilite en effet, la diffusion de l'enzyme vers son substrat (réduction de viscosité de la solution). La formation du coagulum serait favorisée par la régression de l'ionisation des groupements phosphoséryls.

Le maintien de l'aptitude à former un gel sous l'action de la présure du PPCN en dépit d'un traitement thermique intense (100 ${ }^{\circ} \mathrm{C}-5 \mathrm{~min}$ ) (tableau IV) trouve également son origine dans l'élimination par microfiltration-diafiltration de la plus grande partie des protéines solubles. La très faible teneur résiduelle en $\beta-\mathrm{Lg}$ ne permet pas la formation du complexe avec la caséine $\kappa$ (Reddy et Kinsella, 1990). Les sites Phe-Met des formes glycosylées de cette caséine impliquées majoritairement dans la formation du complexe (Ferron-Baumy et al, 1992) restent accessibles à la chymosine.

L'allongement d'environ $20 \%$ du temps de coagulation du PPCN chauffé à $100^{\circ} \mathrm{C}$ - 5 min par rapport au lait cru peut certainement être attribué à un déplacement des équilibres existant entre les formes solubles et colloïdales des sels de calcium. Le calcium précipité lors du chauffage sous la forme d'hydroxyapatite (Evenhuis et de Vries, 1956) se resolubilise peu lors du refroidissement. La solution de PPCN chauffée et refroidie est appauvrie en calcium soluble d'où un retard dans l'apparition du coagulum et l'organisation de ce dernier $\left(K_{20}\right.$ allongé de $65 \%$ et $E_{30}$ inférieur de $32 \%$ à celui du lait cru).

Par ses propriétés de coagulabilité, le PPCN apparaît donc comme une nouvelle matière première particulièrement adaptée à une utilisation en fromagerie, soit comme additif en fromagerie traditionnelle, soit comme produit de base pour la fabrication de caillés ou de fromages nouveaux pouvant même à la limite être réalisés stérilement.

\section{REMERCIEMENTS}

Nous remercions F Remeuf (INAPG, chaire de technologie, Thiverval-Grignon) d'avoir effectué les analyses de tailles micellaires, G Daufin pour d'utiles conseils et pour la lecture du manuscrit.

\section{RÉFÉRENCES}

Acton GH (1977) The determination of lactose in cheese. Aust J Dairy Technol 32, 111-112

Alais C (1984) Sciences du lait. Principes des techniques laitières. Sepaic, Paris, $4^{\mathrm{e}}$ edn

Andrews AT (1983) Proteinases in normal bovine milk and their action on caseins. $J$ Dairy Res 50, 45-55

Bligh EG, Dyer WJ (1959) A rapid method of total lipid extraction and purification. Can $J$ Biochem Physiol 37, 911-917

Brulé G, Fauquant J, Maubois JL (1979) Preparation of native phosphocaseinate by combining membrane ultrafiltration and ultracentrifugation. J Dairy Sci 62, 869-875

Dalgleish DG (1982) The enzymatic coagulation of milk. In: Developments in dairy chemistry 1. Proteins (Fox PF, ed) Appl Sci Publ, Londres

Evenhuis H, de Vries TR (1956) The condition of calcium phosphate in milk III. Neth Milk Dairy J 10, 101-113 
Farrell HM (1988) Physical equilibria: proteins. In: Fundamentals of dairy chemistry (Wong NP, Jenness R, Keeney M, Marth EH, eds) van Nostrand, Reinhold, New York

Fauquant J, Maubois JL, Pierre A (1988) Microfiltration du lait sur membrane minérale. Tech Lait 1028, 21-23

Ferron-Baumy C, Mollé D, Garric G, Maubois JL (1992) Caractérisation des caséinomacropeptides libérés lors de la coagulation par la présure de laits cru et UHT. Lait 72, 165-173

FIL-IDF (1982) Détermination de la teneur en phosphore total dans le fromage. FIL-IDF, norme $n^{\circ} 33 \mathrm{~B}$

FIL-IDF (1988) Détermination de l'indice d'insolubilité. FIL-IDF, norme $n^{\circ} 129 \mathrm{~A}$

Folch J, Lees MB, Sloane-Stanley GH (1957) A simple method for the isolation and purification of the total lipids from animal tissues. $J$ Biol Chem 226, 497-509

Goudédranche $\mathrm{H}$, Maubois $\mathrm{JL}$, Ducruet $\mathrm{P}, \mathrm{Ma}$ haut M (1980) Utilization of the new mineral UF membranes for making semi-hard cheese. Desalination 35, 243-258

Holt C (1985) The milk salts: their secretion, concentrations and physical chemistry. In: Developments in Dairy Chemistry. 3. Lactose and minor constituents (Fox PF, ed) Elsevier, Londres

Ingild A (1983) Single radial immunodiffusion. Scand J Immunol 17, suppl 10, 41-56

Kiviniemi L (1977) Processing of sweet whey and skim milk by ultrafiltration. Thèse: Valio Laboratory Publ, $n^{\circ} 2$, Helsinki, Finlande

Laemmli UK (1970) Cleavage of structural properties during the assembly of the head of bacteriophage T4. Nature (Lond) 227, 680 685

Mangold HK, Kammereck R (1962) New methods of analyzing industrial aliphatic lipids. $J$ Am Oil Chem Soc 39, 201-206

Mati A, Girardet JM, Xenakis D, Linden G (1991) Isolement et caractérisation de la fraction hydrophobe des protéoses peptones des laits bovin, ovin et caprin. Lait 71, 259273

Maubois JL (1984) Separation, extraction and fractionation of milk protein components. Lait $64,485-495$

Mulvihill DM, Fox PF (1989) Physico-chemical and functional properties of milk proteins. In: Developments in dairy chemistry. 4. Functional milk proteins (Fox PF, ed) Elsevier, Londres

Pearce RJ (1983) Thermal separation of $\beta$ lactoglobulin and $\alpha$-lactalbumin in bovine Cheddar cheese whey. Aust J Dairy Technol $38,144-149$

Peri C, Pompei C, Rossi F (1973) Process optimization in skim milk protein recovery and purification by ultrafiltration. J Food Sci 38 , 135-140

Pierre A, Fauquant J (1986) Principes pour un procédé industriel de fractionnement des protéines du lactosérum. Lait 66, 405-419

Reddy M, Kinsella JE (1990) Interaction of $\beta$ lactoglobulin with $\mathrm{K}$-casein in micelles as assessed by chymosin hydrolysis. Effects of added reagents. J Agric Food Chem 38, 366372

Roger L, Maubois JL, Brulé G, Piot M (1979) Procédé pour l'obtention à partir de lactosérum d'un produit enrichi en $\alpha$-lactalbumine et application du dit produit. Brevet Fr 7916 482

Rowland SJ (1938) The determination of the nitrogen distribution in milk. J Dairy Res $9,42-$ 46

Trouvé E, Maubois JL, Piot M, Madec MN, Fauquant J, Rouault A, Tabard J, Brinkman G (1991) Rétention de différentes espèces microbiennes lors de l'épuration du lait par microfiltration en flux tangentiel. Lait 71, 1-13

Webb BH, Johnson AH, Alfort JA (1978) Fundamentals of dairy chemistry. Avi Publishing Co, Westport, 2nd edn 